\title{
Challenges to Tackle Human Immunodeficiency Virus (HIV) Antiretroviral Resistance
}

\author{
Sara Madani ${ }^{1}$, Kaneez Fatima Ishtiaq Qadri ${ }^{2}$ and Ishtiaq Qadri ${ }^{1 *}$ \\ ${ }^{1}$ Department of Biological Sciences, Faculty of Science, King Abdul-Aziz University, Jeddah, KSA \\ ${ }^{2}$ Department of Biological Sciences, Faculty of Science, University of Jeddah, Jeddah, Saudi Arabia
}

*Corresponding Author: Ishtiaq Qadri, Department of Biological Sciences, Faculty of Science, King Abdul-Aziz University, Jeddah, KSA.

E-mail: mqadery@kau.edu.sa

Received: October 22, 2019; Published: November 11, 2019

DOI: 10.31080/ASMI.2019.02.0436

\begin{abstract}
Since 1980s, HIV has been a major health burden on communities and health care system. Treatment options involves combination of Nucleotide Reverse Transcriptase Inhibitors (NRTIs), Non-nucleotide Reverse Transcriptase Inhibitors (NNRTIs), Protease Inhibitors, Integrase Inhibitors and Entry Inhibitors and generally referred to as Antiretroviral Therapy (ART). If proper medication is used at the right stage, it can lower the viral load and risk of viral transmission. However, suboptimal utilization ART is often associated with emergence of drug resistance. Therefore, personalized adherence monitoring and accurate prediction of responses is required to stop the emergence of drug resistant viral strains.

Keywords: HIV (Human Immunodeficiency Virus); Antiretroviral Drugs; Antiretroviral Therapy (ART); Mutations; Drug Susceptibility
\end{abstract}

\section{Introduction}

Human immunodeficiency virus (HIV)) is a retrovirus and belongs to genus lentivirus within the family of Retroviridae [1]. Epidemiologic and phylogenetic analyses available showed that HIV appeared first in Kinshasa, in the Democratic Republic of Congo back in the 1920s, when the virus crosses species line and start infecting humans $[1,2]$.

According to UNAIDS statistics, $\sim 37.9$ million people across the globe with HIV/AIDS and $\sim 1.7$ million became newly infected in 2018, while total deaths related to HIV reaches 0.7 million in 2018 [3]. The first great advance in HIV treatment was in 1987 when AZT (zidovudine) was introduced to the market, other therapy such as the nucleoside analogs ddI (didanosine), ddC (zalcitabine), and d4T (stavudine), these treatments introduced within 1991-1994 period. However, the use of each of them alone as a monotherapy has limited success in patients, and then in September 1995 two nucleoside analogs were introduced instead of one to make it more effective. In June 1996, a third treatment (nevirapine) of non-nucleoside reverse transcriptase inhibitor was introduced. The unscientific term "highly active antiretroviral therapy" (HAART) was used to refer to this cocktails of antiretroviral therapy (ART) [4]. By the end of 2017, 43 anti-retroviral drugs have been approved by US FDA which include 29 single-tablets and 14 fixed-dose combinations (FDCs).
These drugs belong to five different classes of anti-retroviral drugs:

1. Entry inhibitors [5].

2. Integrase inhibitors (INSTIs) [6].

3. Protease inhibitors (PIs) [7].

4. Nucleoside reverse transcriptase inhibitors (NRTIs) [8].

5. Non-nucleoside reverse transcriptase inhibitors (NNRTIs) [9].

In addition, there are a number of fixed-dose combinations (FDCs), including two pharmacoenhancers and four single-tablet regimens (STRs) [10].

There is a challenging necessity to create new class of drugs for multiple reasons such as 1) increasing number of resistance mutations in patients, 2) long-term toxicity of current drugs and problems in adhering to drug regimens. The initiation time of ART therapy is based on multiple bases such as clinical assessment, viral load and CD4 T cell count.

Resistance mutations accumulate in HIV-1 after exposure to antiviral therapy this is causing reduction in the efficacy of treatment, which lead to relapse and treatment failure HIV-1 have several types and subtypes, different subtypes of HIV-1 have different 
consensus sequences in certain sites. That is why every population need to do their own map for drug resistance. This could provide information to develop and enhance the effectiveness of HIV drugs in HIV resistance patients. Sometimes a patient drug resistance profile declares "susceptible to all drugs" and clearly not responding to the drug. This could be due to multiple reasons:

1. Laboratory error.

2. Patient noncompliance to medication.

3. Survival of Minor resistance population of HIV-1.

4. The mutation occur outside the tested region.

5. Drug-Drug interaction.

The database of drug resistance is not updated regularly to include all mutations associated with drug resistance [11]. The updated list of drug resistance mutation is published in 2017 edition by The International Antiviral Society-USA (IAS-USA) but not comprehensive and did not give information about drug susceptibility. For example, 69 insertion is considered major mutation that by itself substantially reduce the effect of all approved Nucleotide Reverse Transcriptase Inhibitors (nRTIs) [11].

In Nonnucleoside Reverse Transcriptase Inhibitors (NNRTIs) class, the following list of mutations are Major mutations that might cause treatment failure to Efavirenz (L100I, K101P, K103N/S, V106M, V108I, Y181C/I, Y188L, G190S/A, P225H, M230L). Mutations that will affect Etravirine drug include (L100I, K101P, Y181C/I/V). Nevirapine major mutations are (L100I, K101P, K103N/S, V106A/M, V108I, Y181C/I, G190A, M230I/L), while Rilpivirine major mutations are ( L100I, K101E/P, E138/A/G/K/Q/R, V179L,Y188L, H221Y, F227C, M230I/L). In protease inhibitor class, Atazanavir and ritonavir major mutations are (I50L, I84V, N88S) [11]. Darunavir/ritonavir major mutations are (I47V, I50V, I54ML, L76V, I84V) the other hand protease inhibitors like ritonavir boosted darunavir have high genetic barrier, so, even when all of these major mutations exist, they will still do their job [12].

According to the latest survey by WHO from 2014-2108, in 18 Asian and African countries, an alarming increase in viral resistance against efavirenz and nevirapine antiretroviral drugs has been observed, particularly among women. Hence, it is recommended to use dolutegravir which is more effective in lowering resistance than efavirenz and nevirapine [13]. The poor medication may have influenced such drug resistance. Any cross resistance could be regulated in the patients by strict adherence/compliance of the drugs and periodic drug-resistance testing.

\section{Bibliography}

1. Luciw PA. "Human immunodeficiency viruses and their replication”. In: Fields BN, editor. Virology. 3rd ed. Philadelphia: Lippincott-Raven (1996): 1881-1952.

2. History of HIV and AIDS overview (2017).

3. https://www.unaids.org/en/resources/fact-sheet

4. WHO: Antiretroviraltherapy (2015).

5. Greenberg ML and Cammack N. "Resistance to enfuvirtide, the first HIV fusion inhibitor". Journal of Antimicrobial Chemotherapy 54 (2004): 333-340.

6. Pandey KK and Grandgenett DP. "HIV-1 Integrase strand transfer inhibitors: Novel insights into their mechanism of action". Retrovirology (2008): 11-16.

7. Hughes PJ., et al. "Protease inhibitors for patients with HIV-1 infection: A comparative overview". Pharmacy and Therapeutics 36.6 (2011): 332-345.

8. Whitcomb JM., et al. "Broad nucleoside reverse-transcriptase inhibitor cross-resistance in human immunodeficiency virus type 1 clinical isolates". The Journal of Infectious Diseases 188 (2003): 992-1000.

9. De Béthune MP. "Non-nucleoside reverse transcriptase inhibitors (NNRTIs), their discovery, development, and use in the treatment of HIV-1 infection: a review of the last 20 years (1989-2009)". Antiviral Research 85 (2010): 75-90.

10. AIDS info Fact Sheet. FDA-Approved HIV Medicines. (2017).

11. Wensing AM., et al. "2017 Update of the drug resistance mutations in HIV-1". Top Antiviral Medicine 24.4 (2016): 132-141.

12. Su TT Chinh., et al. "Structural analyses of 2015-updated drugresistant mutations in HIV-1 protease: an implication of protease inhibitor cross-resistance". BMC Bioinformatics 17.19 (2016): 500.

13. Mega EM. "Alarming Surge in drug-resistance HIV uncovered". Nature News (2019).

Volume 2 Issue 12 December 2019 (C) All rights are reserved by Ishtiaq Qadri., et al. 